\title{
Final comments: looking to the future
}

\author{
Michael Fradley and Nichole Sheldrick
}

We thank Parcak et al. for their response to our paper and for engaging in this important debate, clarifying a number of points regarding their original paper, and raising some important questions about the methods and objectives of the EAMENA project. We can, however, only comment on what was presented in their original article and address, in these brief final remarks, a few of the points raised in their response.

We do not deny that looting is a significant problem, nor are we seeking to downplay the importance of the data that Parcak et al. have collected and their findings with regards to how the threat of looting has developed in the last ten years. Rather, our response critically examined some of the claims made by Parcak et al. that were based on questionable methods, and which, as a result, detracted from their other, valid and important findings.

In particular, their presentation of figures and projection of looting patterns to 2040, even with their caveats, failed to take into account the complexities of the problem and the many unpredictable, human variables that affect looting and the illicit trade in antiquities. As illustrated in Parcak et al.'s example of climate modeling, analyses of these types benefit significantly from the presentation of multiple projections, and these are made stronger when coupled with explicit reasoning as to why specific models have been used and the factors influencing their likelihood. Our own paper therefore offered a few examples simply to illustrate some other possible outcomes, though as they suggest, all of these analyses will need more refining in the future in order to be of use.

Parcak et al. correctly highlight that in the Nile and Delta regions, where their data collection was focused, looting and encroachment, as they define it, are the most significant problems. However, the logic of their assertion that the comparative number of projects working in the Nile and Delta regions versus the Eastern Desert "should demonstrate where most archaeological sites in Egypt exist and are threatened" in general, is flawed. That most projects work on sites in the Nile and Delta only demonstrates where the known sites in the country are, and where academic interest has traditionally been focused. It is for this very reason that EAMENA chose to undertake an investigation of the Eastern Desert and used it as a case study, to invest in, and bring attention to a region that, comparatively speaking, has received limited attention by the heritage community, and, as we demonstrated, is also in urgent need of protection.

Identifying new sites using satellite imagery requires caution, and like most archaeological projects that employ remote sensing techniques, we use well-established methodologies of comparing known sites to new ones in order to assess their likelihood of archaeological significance. In addition, our database employs certainty levels that allow us to indicate our level of confidence in the records that we are creating. Nevertheless, even had we conducted our analyses only taking into account previously published sites, our conclusions would have remained the same: that in the Eastern Desert, the most common and urgent threat in the area is mining. 
It is clear that our projects have taken different approaches to recording and combatting damage to heritage sites. Our criticisms concerning Parcak et al.'s paper and our presentation of the Eastern Desert case study offer an alternative view of the threats facing Egypt's heritage that addressed an imbalance in the work of Parcak et al. This should not detract from the very serious issue of looting and the on-going work being done to prevent it. It is our view that exploring different perspectives and considering alternative interpretations of all our data can only benefit efforts to understand how and why all kinds of damage to heritage occur. What this debate makes clear is the scope for vibrant discussions on these subjects in the future among the wider archaeological community. Ultimately, we are all aiming for the same thing, to help, in whatever ways we can, to preserve, manage, and protect heritage. 